Review

\title{
What Makes A Bacterial Oral Vaccine a Strong Inducer of High-Affinity IgA Responses?
}

\author{
Kathrin Moor ${ }^{\dagger}$ and Emma Slack ${ }^{\dagger}, *$ \\ ETH Zürich, Institute of Microbiology, Vladimir-Prelog-Weg 1-5/10, 8093 Zürich, Switzerland; \\ E-Mail: kathrin.moor@micro.biol.ethz.ch \\ $\dagger$ These authors contributed equally to this work. \\ * Author to whom correspondence should be addressed; E-Mail: emma.slack@micro.biol.ethz.ch; \\ Tel.: +41-44-632-33-55.
}

Academic Editor: Dimiter S. Dimitrov

Received: 7 August 2015 / Accepted: 8 October 2015 / Published: 15 October 2015

\begin{abstract}
Oral vaccination against bacterial pathogens that infect via the gastrointestinal tract is highly desirable for both economic reasons and the supposed benefits of local mucosal immunity. However, the majority of oral vaccine trials in humans result in failure. Here we try to assimilate our current knowledge to generate a model to improve vaccine development strategies. A model previously postulated describes the "immunogenicity" of intestinal bacterial species as a sum of the ability of the species to compete with the microbiota, the "pathogenicity index," and the uniqueness of the species. While this model quite neatly explains the difficulties in generating appropriately attenuated live vaccine strains, it cannot explain the success of fully apathogenic or inactivated high-dose vaccines. We therefore propose a step away from focusing on bacterial traits, and towards the most basic requirements of mucosal vaccines: i.e., the delivery of antigen to the gut-associated lymphoid tissues and the ability of that antigen to induce germinal center formation. While the models seem trivial, both suggest that vaccination strategies permitting uncoupling of disease-causing phenomena from immune stimulation will have a much broader safety margin in a diverse human population. Our modified model further suggests the benefits of delivering antigen in the form of high-dose fully apathogenic or sterile particles, combined with relevant adjuvants.
\end{abstract}


Keywords: oral vaccine; high-affinity intestinal IgA; vaccination strategies; live-attenuation; adjuvant; gut-associated-lymphoid tissue

\section{Introduction}

Most bacterial pathogens enter the body via mucosal surfaces. The mucosal immune system is compartmentalized $[1,2]$, i.e., immune priming at systemic sites does not usually induce strong antibody and $\mathrm{T}$ cell responses at mucosal surfaces and vice versa. This suggests that local priming is preferable for induction of optimal local immune responses. Beyond the advantage of inducing local immunity, oral vaccines are also subject to less stringent regulatory requirements for purification, and are very well suited for rapid mass vaccination as highly trained medical personnel are not needed for application. Nevertheless, currently only two oral vaccines against bacterial infection are licensed for use in humans: Salmonella enterica serovar Typhi (S. Typhi) (Vivotif ${ }^{\mathrm{TM}}$ ) [3] and Vibrio cholerae (V. cholerae) (Dukoral $^{\mathrm{TM}}$, Shanchol ${ }^{\mathrm{TM}}$ ) [4]. This reflects the difficulty of developing effective oral vaccines. Indeed, since the gastrointestinal tract is colonized from birth on with a dense microbial consortium and is repeatedly flooded with food antigens, induction of adaptive immunity in this compartment is necessarily tightly regulated. Peterson and Cardona recently proposed a basic summation model (hereafter referred to as "Peterson and Cardona's basic summation model"), in order to predict the intensity of the adaptive immune response raised against an intestinal microbial species. In this model, the intensity of adaptive immunity is determined by the sum of (a) a bacterial species' capacity to compete with the microbiota, (b) the "pathogenic index", i.e., a sum of the abilities of the microorganisms to harm the host and change the intestinal ecosystem, and (c) its antigenic uniqueness when compared to previously colonizing organisms or self [5]. This crystallizes the principle that has largely guided oral vaccine production, generating strains of pathogens that retain sufficient virulence to compete with the microbiota, but avoid unacceptable pathology in healthy hosts. However, as many mucosal pathogens take advantage of intestinal inflammation to compete with the microbiota [6,7], these behaviors are necessarily linked in live organisms, generating a very narrow therapeutic window. Intriguingly, the Peterson and Cardona's basic summation model cannot account for the success of fully inactivated oral vaccines, such as the currently licensed oral cholera vaccines. Using these concepts as a backbone, we will here try to summarize the current state of our understanding of high-affinity secretory $\operatorname{IgA}(\operatorname{sigA})$ induction in response to oral vaccination and further develop the theoretical framework behind targeted induction of high-affinity sIgA. The issue of whether high-affinity sIgA alone is sufficient to protect from infection is highly pathogen-specific and well reviewed elsewhere [8-10].

\section{Models of Oral Immunogenicity}

\subsection{The Capacity to Compete with the Microbiota and the Pathogenic Index: Attenuation and} Over-Attenuation

The majority of the bacterial oral vaccination field has focused on developing attenuated strains of pathogens or carrier organisms. In recent years, many different strategies to generate safe, live-attenuated 
vaccines have been investigated. Live vaccine strains need to be sufficiently attenuated in order to avoid persistent colonization and induction of unacceptable clinical symptoms; on the other hand, over-attenuation can lead to loss of immunogenicity. This balance is challenging to achieve.

Largely owing to its promise as a vaccine carrier, a large number of rationally attenuated Salmonella vaccine strains have been developed. Similar work on $V$. cholera vaccine strains has been reviewed elsewhere [11,12]. In Salmonella, attenuation is achieved by deletion of a variety of genes encoding for proteins required for either key steps in bacterial metabolism, or signaling and regulation of virulence factors. All strategies result in impaired survival of the vaccine strain after oral administration. The targeted genes encode for proteins involved in aromatic amino acid biosynthesis (aroA, aroC, aroD) [13-17], galactose biosynthesis (galE), heat shock protein ( $h$ tr $A$ ) [18-20], guanine or purine nucleotide biosynthesis (guaA, guaB purA, purB, purE, purH) [21], adenylate cyclase signalling (cya, crp) [22-25], two-component regulatory systems ( $p h o P, p h o Q)$ [26-28], regulators of protein metabolism (clpP, $c l p X)$ [29], outer membrane proteins $(o m p C, o m p F, o m p R)$ [21], and DNA recombination and repair pathways ( $\operatorname{rec} A, \operatorname{rec} B, \operatorname{rec} C)$ [21]. Although many of these studies demonstrated successful vaccination protection in mouse models [21], most of them failed in human clinical trials, often due to unfavorable side effects in healthy volunteers [30-32]. An additional threat with any live-attenuated oral vaccine is the risk to immunocompromised individuals who are vaccinated or, worse still, may be exposed to a vaccine strain shed in the feces or saliva of vaccinated individuals. Attenuated intestinal bacterial pathogens are known to cause severe disease in a range of inherited and acquired immunodeficiencies [33-35]. As logic dictates, considerable effort is now being invested into production of further attenuating mutations and combinations of attenuations. Some recent studies have been performed in immunocompromised mice with superattenuated Salmonella Typhimurium ( $S$. Typhimurium) strains, harboring an additional mutation (e.g., ferric uptake (fur), a putative itaconyl-CoA hydratase (SL1344_3093), antimicrobial resistance (mig-14), or hemolysin expression $(h h a)$ ) on top of the defect in the structural protein type III secretion system 2 ( $\mathrm{sal}$ ) [36-39]. Although it was shown that these superattenuated strains were safer compared to less attenuated vaccine strains, they were still able to colonize tissues and safety was only reported at early time points after application in immunodeficient animals. Thus, frequently the level of attenuation required to induce an immune response is not sufficient to avoid disease.

The reasons for this are reasonably clear from our knowledge of the intestinal and tissue colonization dynamics of non-typhoidal Salmonellae. Virulence factors expressed from Salmonella Pathogenicity Islands 1 and 2 (SPI1 and 2) are required to invade into epithelial cells and survive in phagocytes in the lamina propria [40]. However, only a very small fraction of invading bacteria appear to survive this invasion process [41]. The purpose of this largely suicidal behavior thus appears to be active induction of intestinal inflammation, subsequent shifting of the microbiota composition, and the generation of an optimal intestinal niche for Salmonella replication and transmission [6,41,42]. Any mutation that decreases the functionality of SPI1 and 2 will decrease the virulence and tendency to cause disease, but in the absence of intestinal inflammation, Salmonella competes poorly with the microbiota and cannot reach high numbers in the intestinal lumen or gut-associated lymphoid tissues (GALT) [43].

Interestingly, the licensed human typhoid oral vaccine strain Ty21a was generated many decades ago by non-specific chemical mutagenesis [44] and carries hundreds of point mutations and shifts in genome structure, such that no single mutation can be identified unequivocally as the causative mutation [45]. This strain satisfies the requirements of sufficient colonization to induce high-affinity sIgA in most 
vaccinees with relatively rare pathological sequelae. The question remains: is this the best that can be achieved, and if not, why not?

An unfortunate confounding factor in murine models of Salmonella vaccination and infection is that very low numbers of bacteria, almost regardless of attenuation, enter a persistent, highly resistant state in the GALT [46,47]. This almost permanent colonization can provide adaptive-immunity-independent protection in challenge infection experiments due to niche occupancy [48]. As this is not considered a relevant protective mechanism in the human system, this compounds the problems of translating protection elicited by live-attenuated vaccines in the murine model into human and veterinary medicine.

\subsection{Novel Strategies to Uncouple Competitiveness from Pathology: Auxotrophy and Delayed} Attenuation in Vivo

Curtiss et al. have pioneered strategies to uncouple loss of virulence from loss of competitiveness. They used deletion-insertion mutation strategies to obtain strains that are only virulent in the presence of arabinose, permitting what they refer to as "delayed attenuation." The virulence genes targeted were fur, phoPQ, rpoS, and crp [49]. In vitro culture conditions, where arabinose is supplemented into the media, allow preparation of an inoculum expressing all virulence traits and with the ability to withstand stresses encountered within the gastrointestinal tract (mucin penetration, attachment to epithelium, invasion into epithelium) at early time points post vaccination. Due to the absence of free arabinose in the healthy intestine, the bacteria lost their virulence after a few generations and the vaccinated mice survived even very high vaccination doses.

An alternative uncoupling strategy is regulated lysis of the vaccine strain in vivo [50]. These vaccine strains are able to colonize host lymphoid tissues and induce a robust immune response, but spontaneously lyse after a short period in vivo. To this end, an S. Typhimurium strain has been generated that cannot synthesize the critical cell wall component meso diamino-pimelic acid (m-DAP). In vitro growth is normal in the presence of supplemented m-DAP, but as this supplement is not freely available at high concentrations in vivo, cell wall synthesis is impaired and bacteria lyse, releasing relevant antigen within tissues. m-DAP auxotrophic strains of S. Typhimurium were good inducers of high-affinity sIgA and IgG antibody responses both towards Salmonella outer membrane proteins and to a recombinantly expressed Streptococcus pneumoniae antigen in mice.

These strategies are extremely elegant concepts with the potential to uncouple loss of virulence and loss of competitiveness. Nevertheless, both arabinose and m-DAP are abundant in the intestine within higher-order molecular structures and in some situations, particularly in dysbiosis, free small molecules including sugars can accumulate [51]. Thus the safety of such strains, particularly in individuals with some degree of pre-existing inflammation, remains to be proven. If cross-feeding can be completely avoided, vaccine strains with delayed attenuation and/or lysis in vivo could be a useful strategy to effectively and safely induce mucosal immune responses [52].

\subsection{Can the "Pathogenic Index" Take a Zero Value?}

A postulate of Peterson and Cardona's basic summation model is that in order to achieve immune response competitiveness, pathogenic index and uniqueness must add up to more than the threshold level. This necessarily means that two out of three values could theoretically be zero if the third was 
sufficiently high. As discussed above, live-attenuated vaccine strains are potent inducers of mucosal and systemic antibody responses and many have been shown to be protective in challenge experiments. The reason behind the high potential of live-attenuated vaccines in inducing intestinal high-affinity $\operatorname{sigA}$ responses has been linked to a certain requirement for pathogenicity. Martinoli et al. [2] demonstrated that a high dose of attenuated but invasive S. Typhimurium ( $\triangle$ aroA) can cross M cells and were found in large numbers in Peyer's patches. This invasiveness correlated with the enlargement of the PP, the activation of immune cells, and the release of high-affinity sIgA in the feces. In contrast, although low numbers of noninvasive S. Typhimurium ( $\triangle \operatorname{aroAinvA}$ ) were detected in the PP, they did not induce immune cell activation in this site, and they did not lead to high-affinity sIgA release. Using the nontyphoidal salmonellosis model, Endt et al. demonstrated that acute mucosal inflammation is required to elicit immune responses protecting from enterocolitis [48]. In their work, they demonstrate that when streptomycin pre-treated mice are vaccinated with $5 \times 10^{7}$ avirulent $S$. Typhimurium $(\triangle i n v G s s e D)$ that do not induce intestinal inflammation, no O-antigen specific high-affinity sIgA is induced. Non pre-treated mice inoculated with a low dose of a standard vaccine strain also fail to respond. In these non-pretreated mice, the vaccine strain remains at a density of below $10^{9} \mathrm{cfu} / \mathrm{g}$ feces, which seems to be below the threshold for induction of high-affinity sIgA [53]. In both of these situations, lack of adaptive immunity correlates with rapid, microbiota-dependent clearance of the vaccine strain from the intestine. Thus, at least in the standard live Salmonella system, it appears that some degree of pathogenicity (indeed the induction of some pathology) is essential for the vaccine strain to be able to compete with the intestinal microbiota and grow to a sufficient density/frequency to induce O-antigen specific high-affinity sIgA.

The requirement for a basal level of pathogenicity has also been suggested for enterohemorrhagic E. coli (EHEC). Nagano et al. demonstrated that adhesion of EHEC to intestinal epithelial cells is essential for induction of high-affinity sIgA responses in the intestine. In the absence of virulence factors permitting bacterial adhesion, no high-affinity sIgA was induced. Further, administration of heat-killed EHEC was ignored by the mucosal immune system [54].

In all of these examples, "pathogenicity" has multiple roles. Firstly, as discussed above, induction of inflammation in the intestine tends to be coupled to niche generation for the invading pathogens that are highly resistant to many innate immune defenses [40]. Secondly, damage to host cells and invasion across barriers not normally crossed by microbiota species provide "second signals" for activation of professional antigen-presenting cells. Typically this results in activation of "pattern recognition receptors" and "danger recognition receptors" that increase the expression of co-stimulatory molecules and polarizing cytokines [55]. This should increase the tendency to generate active germinal center reactions in the GALT.

There are, therefore, both good logical reasons why pathogenicity may be required in an oral vaccine, and abundant literature demonstrating that the absence of expression of virulence factors and lack of pathology abrogate immunogenicity. However, in almost all of the systems discussed so far, the authors worked with bacterial vaccine inoculums, typically of fewer than $10^{9}$ bacteria per mouse $[2,48,53,54]$. Using these doses, it appears that the bacteria need to replicate and expand in the intestinal lumen and/or tissues to reach sufficient densities to be recognized by the immune system. It appears that an alternative strategy does exist in which both the pathogenicity and the competitiveness with the microbiota can take zero values. Such a strategy has been meaningfully applied by Hapfelmeier et al. [53]. In this study high levels of high-affinity sIgA against a completely apathogenic E. coli K-12 could be induced in the 
complete absence of pathology if sufficient bacteria were inoculated [53]. Interestingly, using an auxotrophic strain that could not replicate in vivo, they could demonstrate that the dose requirement of more than $10^{9}$ bacteria per mouse was similar in germ-free and colonized mice. This effectively disproves the necessity for pathogenicity in the induction of high-affinity sIgA. If sufficient antigen is inoculated, clearly enough antigen reaches the GALT without any need to compete with the microbiota or induce pathology.

In line with this, the currently available cholera vaccines Shanchol ${ }^{\mathrm{TM}}$ and Dukoral ${ }^{\mathrm{TM}}$ both consist of inactivated whole cells. Shanchol ${ }^{\mathrm{TM}}$ contains three strains of $V$. cholerae $\mathrm{O} 1$ and one of $V$. cholerae $\mathrm{O} 129$ and is orally administered in two doses of $1.75 \times 10^{11}$ cells in a 14-day schedule. Dukoral $^{\mathrm{TM}}$ consists of whole cell $V$. cholerae $\mathrm{O} 1$ plus recombinant cholera toxin B subunit (rCTB) and is administered on a similar schedule. Both vaccines are inactivated by either formalin treatment or heat. The major difference between Shanchol ${ }^{\mathrm{TM}}$ and Dukoral ${ }^{\mathrm{TM}}$ is the presence of $\mathrm{rCTB}$. These vaccines were always assumed to work due to the presence of cholera toxin, either at very low concentrations in the inactivated whole cells or additionally added in recombinant form. The cholera toxin is one of very few known mucosal adjuvants that can promote immunity, also for soluble antigens administered orally [56]. It was claimed that the whole cell preparation has a synergistic effect in combination with recombinant cholera toxin [57]; however, in controlled trials such a synergistic effect could not be demonstrated [58]. In line with the above work from Hapfelmeier et al., the dose of killed bacteria given with these vaccines may actually be sufficient to induce mucosal immunity, without any additional adjuvant. The potential for fully inactivated or completely apathogenic vaccines to be immunogenic, despite identical "uniqueness" to fully virulent strains, suggests that the basic summation model proposed by Peterson and Cardona, while quite appropriate for describing the response to bacterial strains of the microbiota, is not sufficient to guide mucosal vaccine development. A better approximation than "pathogenicity" and "competitiveness" may be to consider the more host-centric concepts of the tendency for antigen to be delivered to GALT and the ability to induce germinal center formation.

It remains to be demonstrated whether IgA responses against high-dose apathogenic vaccines are induced due to very high bacterial antigen loads in the small intestine, which never occur with live-attenuated strains that predominantly expand after arrival in the large intestine [59-61], or if high loads of noninvasive bacterial antigen in the large intestine are sufficient.

\subsection{The Tendency of Antigen to Enter the GALT: Importance of Being Particulate}

The two inactivated cholera vaccines demonstrate that high-affinity SIgA responses can also be induced against sterile antigen. As is necessary for a system to be continuously exposed to high doses of foreign and microbial antigen, the intestinal immune system is rather insensitive to luminal antigens [55]. Therefore, in the absence of tissue invasion or intestinal pathology, a very high concentration of luminal antigen is required for induction of high-affinity $\operatorname{sIgA}$ [53]. A further requirement for the induction of high-affinity sIgA responses seems to be that antigen is delivered in particulate form. Peyer's patches contain $\mathrm{M}$ cells that were shown to take up large antigens such as bacteria, particles, and viruses and deliver them to the underlying follicles to initiate immune responses $[62,63]$. How soluble antigen is sampled in the intestine is not well known, although a recent study by Howe and colleagues implicated a role for a goblet-cell-associated passageway [64]. However, this study did not elucidate a role for 
antigen sampled by this route in adaptive immunity. Indeed, although there are some contradictory studies [65-67], the general dogma is that the feeding of large amounts of soluble antigen leads to the induction of oral tolerance. Oral tolerance has been defined as unresponsiveness to systemic challenge with antigens previously administered orally, e.g., lack of delayed type hypersensitivity reactions and failure to boost systemic IgG responses [68]. This process is thought to play a crucial role in preventing hypersensitivity reactions towards food antigens, but is potentially confounding in oral vaccination.

One of the first demonstrations that particulate antigen was key to intestinal immune stimulation was performed by Macpherson et al. [69]. They could demonstrate that CD103+ DCs carrying commensal bacteria promote protective intestinal high-affinity sIgA responses. Such protective sIgA responses were not induced when mice were treated with split, heat-killed bacteria. Also Hapfelmeier et al. [53] demonstrated that ultraheat-killed bacteria that have lost structural integrity are much less efficient in inducing high-affinity SIgA responses than their reversibly colonizing live bacterial counterparts or peracetic-acid-killed vaccines. However, it remains to be formally established whether particulate antigen is more efficiently sampled, or whether it is less efficiently digested before reaching the GALT.

In order to benefit from the observation that particulate antigen is a more efficient stimulant of the mucosal immune system, several groups are experimenting with the production of conjugated nanoparticles. Howe et al. [70] recently demonstrated that while high doses of soluble OVA administered orally induced oral tolerance, OVA-coated nanoparticles (NP-OVA) induced systemic $\mathrm{IgG1/IgG2c}$ as well as high-affinity sIgA responses. These responses were boosted by subcutaneous injections of OVA + CFA or oral NP-OVA. The description of these prime-boost characteristics are in contrast to Hapfelmeier [53], who described a lack of classical immune memory in his reversible system. This may relate to the nature of the antigen studied (rough E. coli surface structures versus ovalbumin), the nature of the nanoparticles, the mouse system, or other variables not yet considered. It has further been suggested that antigen encapsulation in beta-glucan micro- or nano-particles enhances the mucosal immune response induced after oral application [71]. This effect may be due to the interaction of beta glucans with the pattern recognition receptor Dectin-1, which has also been suggested to play a role in transcytosis by $\mathrm{M}$ cells [72]. The microparticulate nature may also promote more efficient antigen delivery. Inclusion of other M-cell-targeting moieties such as sIgA, specific lectins, or DEC205-binding proteins into nanoparticles may also enhance M cell transcytosis from the gut lumen into the Peyer's patches [73], thus further improving antigen delivery.

\subsection{The Requirement for Oral Adjuvants}

In the blood, spleen, and peripheral lymph nodes, it has long been noted that generation of effective memory responses requires not just antigen, but also adjuvant [74]. In the case of oral vaccines constructed by inactivating whole bacteria, large quantities of ligands for innate immune receptors are necessarily delivered with the bacterial antigens. Nevertheless, these ligands appear to be relatively weak stimuli in the intestinal tract. Hapfelmeier et al., using a fully apathogenic E. coli (that nevertheless contains high concentrations of toll-like receptor ligands and other "pathogen-associated molecular patterns"), observed an additive, rather than synergistic, boosting effect on the high-affinity sIgA response [53]. This would be consistent with poor adjuvanticity of this strategy. Two compounds that demonstrate strong adjuvanticity for the induction of high-affinity $\operatorname{SIgA}$ are cholera toxin (and the closely 
related heat-labile toxin from E. coli) and retinoic acid, a product of vitamin A metabolism. Cholera toxin B subunit, the toxin portion responsible for translocating the catalytic A subunit across the plasma membrane, appears to function by disrupting homeostatic signaling in epithelial cells [75] after binding to surface GM1 receptor. This seems to generate a "danger signal" that translates well into local germinal center formation. The Cholera toxin A subunit, and the corresponding catalytic domain of heat-labile toxin from E. coli, are ADP-ribosylating agents [76]. Considerably greater toxicity is observed when using the holotoxins (A and B subunits together) as adjuvants, albeit associated with increased adjuvant activity [77]. The B subunits alone are therefore more commonly applied as they better balance the induction of immunity with the presence of unacceptable pathology.

In contrast, retinoic acid itself induces the expression of gut-homing addressins [78] and can directly modulate $T$ cell responses [79]. In addition, retinoic acid induces isotype switching to IgA [80,81] and retinoic acid signaling in $\mathrm{B}$ cells has been shown to be essential for induction of antigen-specific high-affinity sIgA responses after oral immunization [82]. Several studies have demonstrated a positive effect of retinoic acid as an adjuvant for vaccination against mucosal infections [82-84]. Interestingly, a recent study has pinpointed Th17 cells that re-differentiate into follicular helper T cells as an important factor for T-cell-dependent sIgA production [85], suggesting that all three of these phenomena may be linked. It should, however, be noted that this paper specifically considered cholera toxin-induced $\operatorname{sigA}$ responses, which may have specific requirements. As retinoic acid also has the huge advantage of being a vitamin derivative with no negative associations - in contrast to cholera toxin, which is hard to sell to the general public - this is likely to be heavily investigated as a potential oral adjuvant for medical use.

\subsection{The Tendency of Antigen to Reach GALT: The Role of Pre-existing Inflammation, Microbiota} Composition, and Age

Oral vaccines have been shown to induce diverse levels of seroconversion. The most prominent examples are the failure of the oral polio vaccine in India [86] and the rotavirus vaccine in Africa [87,88], whereas both vaccines confer good protection in developed countries. There is increasing evidence that the state of the host significantly affects the success of oral vaccines, much more so than for parenteral vaccines. Several clinical trials have demonstrated diminished immune responses and/or lower vaccine efficacy of oral vaccines in developing countries, where many mucosal pathogens are endemic [89]. It is very likely that several factors lead to this phenomenon. There appears to be a nutritional factor, including uptake of calories but also micronutrients such as vitamin A [90,91] and zinc [92]. In addition, pre-existing intestinal inflammation (chronic environmental enteropathy) is suspected to be a leading cause of the failure of oral vaccines in people living under poor sanitation and hygiene conditions [93,94]. In endemic regions, it has also been hypothesized that transfer of maternal antibodies present due to recent infection (via placenta or breast milk) could negatively influence the potential of oral vaccines to induce immune responses in young children [89].

Host genetics will also influence the efficacy of an oral vaccine. In a recent study, Majumder et al. correlated variation in seroconversion to an oral cholera vaccine to host genetic differences [95]. Such genetic variations are impossible to assess when oral vaccines are studied in single inbred animal models, but should be accounted for in large-scale clinical trials. 
Besides pathogenic infection and genetic factors, there is strong evidence that the host microbiota influence the responsiveness to oral vaccines. Generally, highly complex and stable microbiota compositions lower the probability of an incoming novel species (i.e., a live-attenuated vaccine strain) establishing a niche and successfully colonizing the intestine [96]. This so-called "colonization resistance" protects the host from enteric pathogens, but it can also prevent the stable colonization of a liveattenuated vaccine strain. Interestingly, the microbiota can also have more global effects on intestinal sIgA levels. Moon et al. [97] demonstrated that certain species in the intestinal microbiota can degrade the secretory component of sIgA and thereby reduce the levels of total intestinal sIgA. Further, there are numerous studies suggesting that antibiotic treatment either enhances or inhibits the response to oral vaccines [98-100]. The variation observed may be due to a highly complex interplay between live-attenuated strains that are differentially susceptible to antibiotics, differential depletion of microbiota constituents, and direct side-effects of certain antibiotics on mammalian cells. Of note, Hapfelmeier et al. [53] found a very similar dose requirement using a non-replicating apathogenic E. coli vaccine in germ-free mice to that observed in fully colonized mice [69], but colonization was associated with rapid attrition of the specific sIgA response. Thus the interaction of the microbiota with intestinal vaccination is potentially highly complex: colonization resistance can prevent sufficient densities of live-attenuated vaccine strains establishing in the intestinal lumen; certain microbiota species can rapidly degrade sIgA; and via interaction with the mucosal immune system, different species may promote or inhibit antigen presentation and signaling induced by mucosal vaccines, and particular species may be associated with faster attrition of mucosal sIgA responses.

Besides having an effect on the levels of total $\operatorname{sIgA}$, the microbiota also shape the sIgA repertoire during an individual's life. Lindner et al. recently investigated what factors shape the intestinal sIgA repertoire and how stable this system is over time [101]. They demonstrated that each individual harbors a sIgA repertoire that is unique, highly diverse, and polyclonal. Further, they showed that with increasing age, the sIgA repertoire gained diversity and, in the murine model, the total intestinal sIgA levels increase continuously with age.

Together, this raises critical issues in vaccine development. It is absolutely essential that microbiota composition is well controlled in pre-clinical studies to avoid artifacts due to potent effects on the tendency of a vaccine strain to colonize, on the rate of high-affinity sIgA degradation, and on the "natural" IgA repertoire. Perhaps more challenging is that, in most cases, an oral vaccine is pressingly required specifically for the demographic known to respond poorly. It would therefore be highly beneficial for oral vaccine research to develop an animal model that can mimic the persistent infectious burden and nutritional status present in endemic regions and variants of the model that carry some genetic diversity.

\section{7. "Uniqueness": Is There Evidence for Cross-Tolerance and Immunodominance in the Intestine?}

The final postulate of the Peterson and Cardona basic summation model is that an organism will be more antigenic if it is more different from microorganisms previously experienced. It is clear that regulatory $\mathrm{T}$ cells and the ability of $\mathrm{T}$ cells to respond to the cytokine IL-10 are essential to maintain homeostasis in the intestinal immune system [102,103], suggesting an important role in the decision to mount adaptive immune responses. Some degree of Treg specificity for the microbiota is required in 
order to suppress pathological inflammation induced by the microbiota [104]. However, it remains unclear how important this is in preventing the induction of high-affinity sIgA responses. Some bacterial antigens, in particular the proteins making up the large and small ribosomal subunits, are more than $90 \%$ conserved even between phyla in the intestine, suggesting that some antigenic overlap will always exist. Further, Linder et al. demonstrate recruitment of a new subpopulation of B cells after infection with a live-attenuated Salmonella vaccine strain, with minimal changes in the overall plasma cell community, indicating that despite the likely presence of cross-reactive antigen in the microbiota, this does not trigger a rapid memory or immunodominant response [105]. The issue of "uniqueness" may become more important in the case of vaccine carrier strains, where pre-existing immunity against the carrier may prevent induction of any immunity against the recombinant antigen [106,107]. Additionally CTB, when used as an adjuvant, clearly induces antibodies against itself, potentially eliminating its adjuvant effect in subsequent vaccinations.

\section{Conclusions: The Perfect Oral Vaccine}

In conclusion, the field of oral vaccination against bacterial infection has been heavily focused on developing live-attenuated strains that attempt to optimize the amount of virulence, such that the sum of the remaining "pathogenicity" plus the remaining "competitiveness" is above the required threshold to induce immunity. Given the important effect of the microbiota and chronic infection on the sensitivity of the intestinal immune system, we would suggest that this will always be challenging to tailor to a level suitable for the entire human population. Delayed attenuation and auxotrophy offer much greater tunability, but with the inherent problems of cross-feeding. As high doses of antigen in the complete absence of virulence or competition can clearly induce high levels of high-affinity sIgA, we would suggest that a more trivial model based on host factors clearly critical for antibody responses is actually more informative for vaccine design.

In this simplified model, immunogenicity can be defined as a complex product of the tendency of antigen to reach the GALT, the tendency to induce full germinal center reactions, the sensitivity of the host's mucosal immune system due to microbiota composition or infection, and (in the case of vaccine carriers or antigenic adjuvants) the uniqueness of the vaccination antigen cocktail. As zero antigen reaching the GALT and zero induction of germinal centers are both incompatible with generation of adaptive immunity, the model cannot be described with simple summation. The relationship between production of high-affinity sIgA, antigen delivery to GALT and induction of germinal center reactions is roughly sketched in Figure 1. 


\section{A. Peterson and Cardona model}

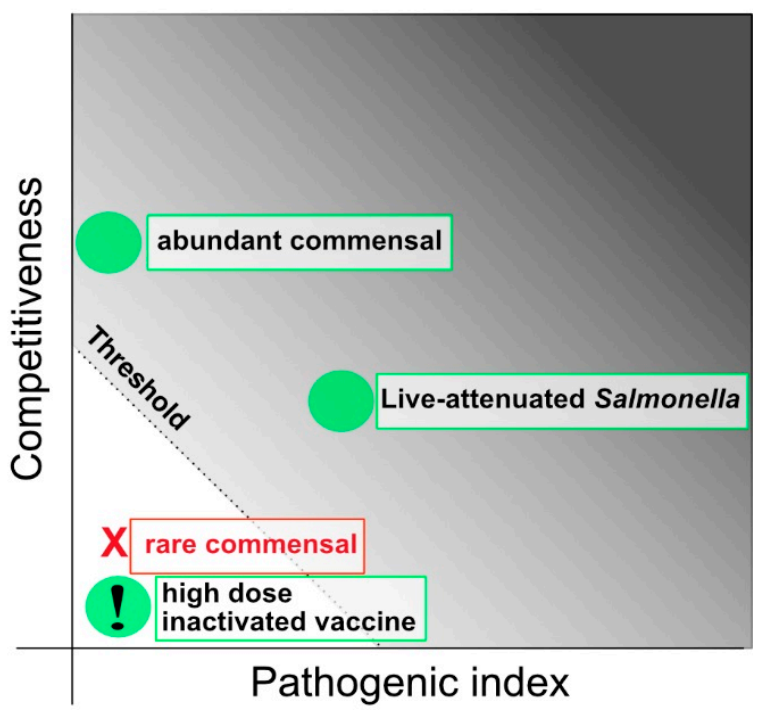

B. The product model

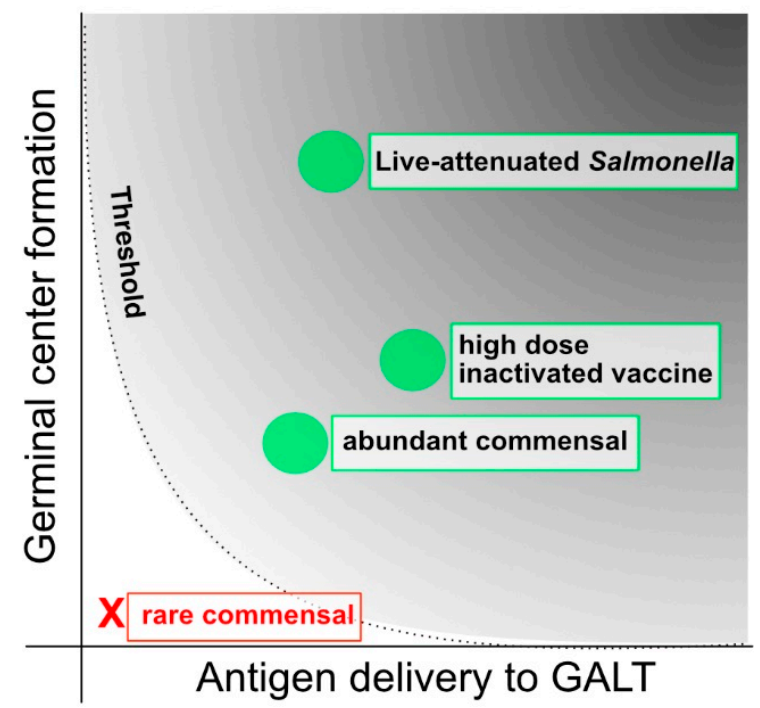

Figure 1. Theoretical models of high-affinity sIgA induction. (A) Theoretical depiction of Peterson and Cardona's basic summation model based on competition with the microbiota and pathogenicity versus (B) one based on GALT entry and germinal center formation. Gray shading indicates the areas where high-affinity sIgA responses would be expected to be elicited. Entities known to elicit strong high-affinity sIgA responses are shown with green circles. "!" denotes entities that are incompatible with the model's predictions. Entities eliciting poor or no antibody responses are shown with a red "X." High-dose inactivated vaccine behavior can only be explained by the adapted model (B).

It naturally follows that a live bacterium that can grow to high densities in the intestinal lumen by competing with the microbiota is more likely to be sampled into the GALT. Pathogenicity that decreases the barrier function of the intestinal epithelium will also increase this parameter. Thus live-attenuated Salmonella vaccine strains fall into the area of the graph expected to generate high-affinity sIgA responses in both models (Figure 1). However, very high doses of sterile particulate antigen, such as the licensed cholera vaccines, have a high value for GALT antigen delivery, but very low values for both pathogenicity and competitiveness with the microbiota. Thus, they would be expected to fail from the basic summation model proposed by Peterson and Cardona, but to fall within the permissive area when GALT entry and ability to induce germinal centers is evoked (Figure 1). The induction of germinal center reactions requires the presence of signals that activate $\mathrm{T}$ cells and overcome immune regulatory signals. Classical "pathogen-associated molecular patterns" in very high concentrations in the lamina propria may be just sufficient to achieve this, but are clearly inefficient [53]. However, pathogenicity is likely to deliver a much stronger set of signals that promote germinal center formation along with inflammation. Thus, these two terms extend the parameters suggested by Peterson and Cardona to also describe high-affinity sIgA responses against sterile particulate antigen. Clearly much remains to be understood. A gaping hole in the oral vaccination tool kit is that of appropriate well-tested oral adjuvants that would permit the construction of optimal sterile vaccines. Additionally, we have only considered the factors driving high-affinity SIgA responses to build our extended model. It is rather naive to think that an oral vaccine's success depends only on its ability to induce high-affinity sIgA-local and 
systemic $\mathrm{T}$ cell responses, as well as systemic antibody responses, may be critical for protection and may have more specific requirements for their generation. Further, the precise antigenic targets of the response will determine whether it is protective or not for each individual pathogen under consideration. Future research on the ability of oral vaccines, or combined parenteral and oral vaccination strategies, to induce relevant T-cell-mediated immunity would allow us to extend this simplified concept of oral immunogenicity to a more comprehensive response. Nevertheless, the modified model points to promising avenues for future oral vaccine research. Standard live-attenuation strategies require hitting a "sweet spot" in the level of attenuation to satisfy the criteria of absence of severe pathology and sufficient immune activation. Given the variation in the human population due to genetics, microbiota composition, and co-infection, this will be almost impossible to achieve at the population level. Development of safe delayed-attenuation or auxotrophic systems would provide a useful alternative, but current strategies carry a high risk of cross-feeding. Use of high-dose apathogenic vaccines, preferably combined with relevant oral adjuvants, appears to be an under-researched area, presumably due to the false idea that sterile antigen is ineffective. However, such strategies have been demonstrated to be effective and completely safe in several models and in two clinically relevant vaccines. Finally, the challenge posed by bacterial vaccine failure in endemic regions of the world needs to be addressed and relevant models for pre-clinical vaccine testing developed to assess the effects of chronic infection and malnutrition on vaccine efficacy.

\section{Acknowledgments}

We thank Wolf-Dietrich Hardt for his critical reading of the manuscript.

\section{Author Contributions}

Kathrin Moor and Emma Slack wrote the review with equal contribution.

\section{Conflicts of Interest}

The authors declare no conflict of interest.

\section{References}

1. Macpherson, A.J.; Uhr, T. Compartmentalization of the mucosal immune responses to commensal intestinal bacteria. Ann. NY Acad. Sci. 2004, 1029, 36-43.

2. Martinoli, C.; Chiavelli, A.; Rescigno, M. Entry route of Salmonella typhimurium directs the type of induced immune response. Immunity 2007, 27, 975-984.

3. Date, K.A.; Bentsi-Enchill, A.; Marks, F.; Fox, K. Typhoid fever vaccination strategies. Vaccine 2015, 33 (Suppl. 3), C55-C61.

4. Kabir, S. Critical analysis of compositions and protective efficacies of oral killed cholera vaccines. Clin. Vaccine Immunol. 2014, 21, 1195-1205.

5. Peterson, D.A.; Cardona, R.A. Specificity of the adaptive immune response to the gut microbiota. Adv. Immunol. 2010, 107, 71-107. 
6. Stecher, B.; Robbiani, R.; Walker, A.W.; Westendorf, A.M.; Barthel, M.; Kremer, M.; Chaffron, S.; Macpherson, A.J.; Buer, J.; Parkhill, J.; et al. Salmonella enterica serovar typhimurium exploits inflammation to compete with the intestinal microbiota. PLoS Biol. 2007, 5, 2177-2189.

7. Lupp, C.; Robertson, M.L.; Wickham, M.E.; Sekirov, I.; Champion, O.L.; Gaynor, E.C.; Finlay, B.B. Host-mediated inflammation disrupts the intestinal microbiota and promotes the overgrowth of Enterobacteriaceae. Cell Host Microbe 2007, 2, 119-129.

8. Sztein, M.B.; Salerno-Goncalves, R.; McArthur, M.A. Complex adaptive immunity to enteric fevers in humans: lessons learned and the path forward. Front. Immunol. 2014, 5, 516.

9. Bishop, A.L.; Camilli, A. Vibrio cholerae: Lessons for mucosal vaccine design. Expert Rev. Vaccines 2011, 10, 79-94.

10. Franco, M.A.; Angel, J.; Greenberg, H.B. Immunity and correlates of protection for rotavirus vaccines. Vaccine 2006, 24, 2718-2731.

11. Viret, J.F.; Dietrich, G.; Favre, D. Biosafety aspects of the recombinant live oral Vibrio cholerae vaccine strain CVD 103-HgR. Vaccine 2004, 22, 2457-2469.

12. Ryan, E.T.; Calderwood, S.B.; Qadri, F. Live attenuated oral cholera vaccines. Expert Rev. Vaccines 2006, 5, 483-494.

13. Hoiseth, S.K.; Stocker, B.A. Aromatic-dependent Salmonella typhimurium are non-virulent and effective as live vaccines. Nature 1981, 291, 238-239.

14. Eisenstein, T.K.; Killar, L.M.; Stocker, B.A.; Sultzer, B.M. Cellular immunity induced by avirulent Salmonella in LPS-defective C3H/HeJ mice. J. Immunol. 1984, 133, 958-961.

15. Killar, L.M.; Eisenstein, T.K. Immunity to Salmonella typhimurium infection in $\mathrm{C} 3 \mathrm{H} / \mathrm{HeJ}$ and $\mathrm{C} 3 \mathrm{H} / \mathrm{HeNCrlBR}$ mice: Studies with an aromatic-dependent live S. typhimurium strain as a vaccine. Infect. Immun. 1985, 47, 605-612.

16. Harrison, J.A.; Villarreal-Ramos, B.; Mastroeni, P.; Demarco de Hormaeche, R.; Hormaeche, C.E. Correlates of protection induced by live Aro- Salmonella typhimurium vaccines in the murine typhoid model. Immunology 1997, 90, 618-625.

17. Hormaeche, C.E.; Mastroeni, P.; Harrison, J.A.; Demarco de Hormaeche, R.; Svenson, S.; Stocker, B.A.D. Protection against oral challenge three months after i.v. immunization of BALB/c mice with live Aro Salmonella typhimurium and Salmonella enteritidis vaccines is serotype (species)dependent and only partially determined by the main LPS O antigen. Vaccine 1996, 14, 251-259.

18. Chatfield, S.N.; Strahan, K.; Pickard, D.; Charles, I.G.; Hormaeche, C.E.; Dougan, G. Evaluation of Salmonella typhimurium strains harbouring defined mutations in htrA and aroA in the murine salmonellosis model. Microb. Pathog. 1992, 12, 145-151.

19. Lowe, D.C.; Savidge, T.C.; Pickard, D.; Eckmann, L.; Kagnoff, M.F.; Dougan, G.; Chatfield, S.N. Characterization of candidate live oral Salmonella typhi vaccine strains harboring defined mutations in aroA, aroC, and htrA. Infect. Immun. 1999, 67, 700-707.

20. Tacket, C.O.; Sztein, M.B.; Losonsky, G.A.; Wasserman, S.S.; Nataro, J.P.; Edelman, R.; Pickard, D.; Dougan, G.; Chatfield, S.N.; Levine, M.M. Safety of live oral Salmonella typhi vaccine strains with deletions in htrA and aroC aroD and immune response in humans. Infect. Immun. 1997, 65, $452-456$. 
21. Simon, R.; Tennant, S.M.; Galen, J.E.; Levine, M.M. Mouse models to assess the efficacy of non-typhoidal Salmonella vaccines: Revisiting the role of host innate susceptibility and routes of challenge. Vaccine 2011, 29, 5094-5106.

22. Curtiss, R., 3rd; Kelly, S.M. Salmonella typhimurium deletion mutants lacking adenylate cyclase and cyclic AMP receptor protein are avirulent and immunogenic. Infect. Immun. 1987, 55, 3035-3043.

23. Coe, N.E.; Wood, R.L. The effect of exposure to a delta cya/delta crp mutant of Salmonella typhimurium on the subsequent colonization of swine by the wild-type parent strain. Vet. Microbiol. 1992, 31, 207-220.

24. Hassan, J.O.; Curtiss, R., 3rd. Control of colonization by virulent Salmonella typhimurium by oral immunization of chickens with avirulent delta cya delta crp S. typhimurium. Res. Microbiol. 1990, $141,839-850$.

25. Hassan, J.O.; Curtiss, R., 3rd. Effect of vaccination of hens with an avirulent strain of Salmonella typhimurium on immunity of progeny challenged with wild-Type Salmonella strains. Infect. Immun . 1996, 64, 938-944.

26. Galan, J.E.; Curtiss, R., 3rd. Virulence and vaccine potential of phoP mutants of Salmonella typhimurium. Microb. Pathog. 1989, 6, 433-443.

27. Hohmann, E.L.; Oletta, C.A.; Miller, S.I. Evaluation of a phoP/phoQ-deleted, aroA-deleted live oral Salmonella typhi vaccine strain in human volunteers. Vaccine 1996, 14, 19-24.

28. Matsui, H.; Isshiki, Y.; Eguchi, M.; Ogawa, Y.; Shimoji, Y. Evaluation of the live vaccine efficacy of virulence plasmid-cured, and phoP- or aroA-deficient Salmonella enterica serovar Typhimurium in mice. J. Vet. Med. Sci. 2015, 77, 181-186.

29. Matsui, H.; Suzuki, M.; Isshiki, Y.; Kodama, C.; Eguchi, M.; Kikuchi, Y.; Motokawa, K.; Takaya, A.; Tomoyasu, T.; Yamamoto, T. Oral immunization with ATP-dependent protease-deficient mutants protects mice against subsequent oral challenge with virulent Salmonella enterica serovar typhimurium. Infect. Immun. 2003, 71, 30-39.

30. Dilts, D.A.; Riesenfeld-Örn, I.; Fulginiti, J.P.; Ekwall, E.; Granert, C.; Nonenmacher, J.; Brey, R.N.; Cryz, S.J.; Karlsson, K.; Bergman, K.; et al. Phase I clinical trials of aroA aroD and aroA aroD htrA attenuated S. typhi vaccines; effect of formulation on safety and immunogenicity. Vaccine 2000, 18, 1473-1484.

31. Hone, D.M.; Tacket, C.O.; Harris, A.M.; Kay, B.; Losonsky, G.; Levine, M.M. Evaluation in volunteers of a candidate live oral attenuated Salmonella typhi vector vaccine. J. Clin. Invest. 1992, 90, 412-420.

32. Tacket, C.O.; Hone, D.M.; Curtiss, R., 3rd; Kelly, S.M.; Losonsky, G.; Guers, L.; Harris, A.M.; Edelman, R.; Levine, M.M. Comparison of the safety and immunogenicity of delta aroC delta aroD and delta cya delta crp Salmonella typhi strains in adult volunteers. Infect. Immun. 1992, 60, 536-541.

33. Casanova, J.L.; Jouanguy, E.; Lamhamedi, S.; Blanche, S.; Fischer, A. Immunological conditions of children with BCG disseminated infection. Lancet 1995, 346, 581.

34. Hesseling, A.C.; Rabie, H.; Marais, B.J.; Manders, M.; Lips, M.; Schaaf, H.S.; Gie, R.P.; Cotton, M.F.; van Helden, P.D.; Warren, R.M.; et al. Bacille Calmette-Guerin vaccine-induced disease in HIV-infected and HIV-uninfected children. Clin. Infect. Dis. 2006, 42, 548-558. 
35. Felmy, B.; Songhet, P.; Slack, E.M.C.; Müller, A.J.; Kremer, M.; Van Maele, L.; Cayet, D.; Heikenwalder, M.; Sirard, J.-C.; Hardt, W.D. NADPH oxidase deficient mice develop colitis and bacteremia upon infection with normally avirulent, TTSS-1- and TTSS-2-deficient Salmonella Typhimurium. PLOS ONE 2013, 8, e77204.

36. Vishwakarma, V.; Pati, N.B.; Chandel, H.S.; Sahoo, S.S.; Saha, B.; Suar, M. Evaluation of Salmonella enterica serovar Typhimurium TTSS-2 deficient fur mutant as safe live-attenuated vaccine candidate for immunocompromised mice. PLOS ONE 2012, 7, e52043.

37. Periaswamy, B.; Maier, L.; Vishwakarma, V.; Slack, E.; Kremer, M.; Andrews-Polymenis, H.L.; McClelland, M.; Grant, A.J.; Suar, M.; Hardt, W.-D. Live attenuated S. Typhimurium vaccine with improved safety in immuno-compromised mice. PLoS ONE 2012, 7, e45433.

38. Pati, N.B.; Vishwakarma, V.; Selvaraj, S.K.; Dash, S.; Saha, B.; Singh, N.; Suar, M. Salmonella Typhimurium TTSS-2 deficient mig-14 mutant shows attenuation in immunocompromised mice and offers protection against wild-type Salmonella Typhimurium infection. BMC Microbiol. 2013, $13,236$.

39. Vishwakarma, V.; Pati, N.B.; Ray, S.; Das, S.; Suar, M. TTSS2-deficient hha mutant of Salmonella Typhimurium exhibits significant systemic attenuation in immunocompromised hosts. Virulence 2014, 5, 311-320.

40. Kaiser, P.; Diard, M.; Stecher, B.; Hardt, W.-D. The streptomycin mouse model for Salmonella diarrhea: functional analysis of the microbiota, the pathogen's virulence factors, and the host's mucosal immune response. Immunol. Rev. 2012, 245, 56-83.

41. Ackermann, M.; Stecher, B.; Freed, N.E.; Songhet, P.; Hardt, W.-D.; Doebeli, M. Self-destructive cooperation mediated by phenotypic noise. Nature 2008, 454, 987-990.

42. Diard, M.; Garcia, V.; Maier, L.; Remus-Emsermann, M.N.P.; Regoes, R.R.; Ackermann, M.; Hardt, W.-D. Stabilization of cooperative virulence by the expression of an avirulent phenotype. Nature 2013, 494, 353-356.

43. Stecher, B.; Hardt, W.D. Mechanisms controlling pathogen colonization of the gut. Curr. Opin. Microbiol. 2011, 14, 82-91.

44. Germanier, R.; Fuer, E. Isolation and characterization of Gal E mutant Ty 21a of Salmonella typhi: a candidate strain for a live, oral typhoid vaccine. J. Infect. Dis. 1975, 131, 553-558.

45. Xu, D.; Cisarb, J.O.; Polyc, F.; Yangb, J.; Albanesea, J.; Dharmasenaa, M.; Waia, T.; Guerryc, P.; Kopeckoa, D.J. Genome Sequence of Salmonella enterica Serovar Typhi Oral Vaccine Strain Ty21a. Genome Announc. 2013, 1, e00650-13.

46. Kaiser, P.; Regoes, R.R.; Dolowschiak, T.; Wotzka, S.Y.; Lengefeld, J.; Slack, E.; Grant, A.J.; Ackermann, M.; Hardt, W.-D. Cecum lymph node dendritic cells harbor slow-growing bacteria phenotypically tolerant to antibiotic treatment. PLoS Biol. 2014, 12, e1001793.

47. Grant, A.J.; Morgan, F.J.E.; McKinley, T.J.; Foster, G.L.; Maskell, D.J.; Mastroeni, P. Attenuated Salmonella Typhimurium lacking the pathogenicity island-2 type 3 secretion system grow to high bacterial numbers inside phagocytes in mice. PLoS Pathog. 2012, 8, e1003070.

48. Endt, K.; Stecher, B.; Chaffron, S.; Slack, E.; Tchitchek, N.; Benecke, A.; Van Maele, L.; Sirard, J.-C.; Mueller, A.J.; Heikenwalder, M.; et al. The microbiota mediates pathogen clearance from the gut lumen after non-typhoidal Salmonella diarrhea. PLoS Pathog. 2010, 6, e1001097. 
49. Curtiss, R., 3rd; Wanda, S.-Y.; Gunn, B.M.; Zhang, X.; Tinge, S.A.; Ananthnarayan, V.; Mo, H.; Wang, S.; Kong, W. Salmonella enterica serovar typhimurium strains with regulated delayed attenuation in vivo. Infect. Immun. 2009, 77, 1071-1082.

50. Kong, W.; Wanda, S.-Y.; Gunn, B.M.; Zhang, X.; Tinge, S.A.; Ananthnarayan, V.; Mo, H.; Wang, S.; Kong, W. Regulated programmed lysis of recombinant Salmonella in host tissues to release protective antigens and confer biological containment. Proc. Natl. Acad. Sci. USA 2008, 105, 9361-9366.

51. Ng, K.M.; Ferreyra, J.A.; Higginbottom, S.K.; Lynch, J.B.; Kashyap, P.C.; Gopinath, S.; Naidu, N.; Choudhury, B.; Weimer, B.C.; Monack, D.M.; et al. Microbiota-liberated host sugars facilitate post-antibiotic expansion of enteric pathogens. Nature 2013, 502, 96-99.

52. Wang, S.; Curtiss, R., 3rd. Development of Vaccines Using Live Vectors. Vaccines (Basel) 2014, 2, 49-88.

53. Hapfelmeier, S.; Lawson, M.A.E.; Slack, E.; Kirundi, J.K.; Stoel, M.; Heikenwalder, M.; Cahenzli, J.; Velykoredko, Y.; Balmer, M.L.; Endt, K.; et al. Reversible microbial colonization of germ-free mice reveals the dynamics of IgA immune responses. Science 2010, 328, 1705-1709.

54. Nagano, K.; Taguchi, K.; Tokoro, S.; Tatsuno, I.; Mori, H. Adhesion of enterohemorrhagic Escherichia coli O157:H7 to the intestinal epithelia is essential for inducing secretory IgA antibody production in the intestine of mice. Biol. Pharm. Bull. 2014, 37, 409-416.

55. Garside, P.; Millington, O.; Smith, K.M. The anatomy of mucosal immune responses. Ann. NY Acad. Sci. 2004, 1029, 9-15.

56. Langridge, W.; Denes, B.; Fodor, I. Cholera toxin B subunit modulation of mucosal vaccines for infectious and autoimmune diseases. Curr. Opin. Investig. Drugs 2010, 11, 919-928.

57. Svennerholm, A.M.; Holmgren, J. Synergistic protective effect in rabbits of immunization with Vibrio cholerae lipopolysaccharide and toxin/toxoid. Infect. Immun. 1976, 13, 735-740.

58. Black, R.E.; Levine, M.M.; Clements, M.L.; Young, C.R.; Svennerholm, A.M.; Holmgren, J. Protective efficacy in humans of killed whole-vibrio oral cholera vaccine with and without the B subunit of cholera toxin. Infect. Immun. 1987, 55, 1116-1120.

59. Barthel, M.; Hapfelmeier, S.; Quintanilla-Martínez, L.; Kremer, M.; Rohde, M.; Hogardt, M.; Pfeffer, K.; Rüssmann, H.; Hardt, W.-D. Pretreatment of mice with streptomycin provides a Salmonella enterica serovar Typhimurium colitis model that allows analysis of both pathogen and host. Infect. Immun. 2003, 71, 2839-2858.

60. Hapfelmeier, S.; Hardt, W.D. A mouse model for S. typhimurium-induced enterocolitis. Trends Microbiol. 2005, 13, 497-503.

61. Hapfelmeier, S.; Ehrbar, K.; Stecher, B.; Barthel, M.; Kremer, M.; Hardt, W.-D. Role of the Salmonella pathogenicity island 1 effector proteins SipA, SopB, SopE, and SopE2 in Salmonella enterica subspecies 1 serovar Typhimurium colitis in streptomycin-pretreated mice. Infect. Immun. 2004, 72, 795-809.

62. Neutra, M.R.; Mantis, N.J.; Kraehenbuhl, J.P. Collaboration of epithelial cells with organized mucosal lymphoid tissues. Nat. Immunol. 2001, 2, 1004-1009.

63. Mabbott, N.A.; Donaldson, D.S.; Ohno, H.; Williams, I.R.; Mahajan, A. Microfold (M) cells: Important immunosurveillance posts in the intestinal epithelium. Mucosal Immunol. 2013, 6, $666-677$. 
64. Howe, S.E.; Lickteig, D.J.; Plunkett, K.N.; Ryerse, J.S.; Konjufca, V. The Uptake of Soluble and Particulate Antigens by Epithelial Cells in the Mouse Small Intestine. PLoS ONE 2014, 9, doi:10.1371/journal.pone.0086656.

65. Strindelius, L.; Degling Wikingsson, L.; Sjoholm, I. Extracellular antigens from Salmonella enteritidis induce effective immune response in mice after oral vaccination. Infect. Immun. 2002, 70, 1434-1442.

66. Strindelius, L.; Filler, M.; Sjoholm, I. Mucosal immunization with purified flagellin from Salmonella induces systemic and mucosal immune responses in $\mathrm{C} 3 \mathrm{H} / \mathrm{HeJ}$ mice. Vaccine 2004, 22 , 3797-3808.

67. Strindelius, L.; Folkesson, A.; Normark, S.; Sjöholm, I. Immunogenic properties of the Salmonella atypical fimbriae in BALB/c mice. Vaccine 2004, 22, 1448-1456.

68. Weiner, H.L.; da Cunha, A.P.; Quintana, F.; Wu, H. Oral tolerance. Immunol. Rev. 2011, 241, 241-259.

69. Macpherson, A.J.; Uhr, T. Induction of protective IgA by intestinal dendritic cells carrying commensal bacteria. Science 2004, 303, 1662-1665.

70. Howe, S.E.; Konjufca, V.H. Per-oral immunization with antigen-conjugated nanoparticles followed by sub-cutaneous boosting immunization induces long-lasting mucosal and systemic antibody responses in mice. PLOS ONE 2015, 10, e0118067.

71. De Smet, R.; Demoor, T.; Verschuere, S.; Dullaers, M.; Ostroff, G.R.; Leclercq, G.; Allais, L.; Pilette, C.; Dierendonck, M.; De Geest, B.G.; et al. beta-Glucan microparticles are good candidates for mucosal antigen delivery in oral vaccination. J. Control. Release 2013, 172, 671-678.

72. Rochereau, N.; Drocourt, D.; Perouzel, E.; Pavot, V.; Redelinghuys, P.; Brown, G.D.; Tiraby, G.; Roblin, X.; Verrier, B.; Genin, C.; et al. Dectin-1 is essential for reverse transcytosis of glycosylated SIgA-antigen complexes by intestinal M cells. PLoS Biol. 2013, 11, e1001658.

73. Vyas, S.P.; Gupta, P.N. Implication of nanoparticles/microparticles in mucosal vaccine delivery. Expert Rev. Vaccines 2007, 6, 401-418.

74. Janeway, C.A., Jr. Approaching the asymptote? Evolution and revolution in immunology. Cold Spring Harb. Symp. Quant. Biol. 1989, 54 (Pt. 1), 1-13.

75. Glenn, G.M.; Francis, D.H.; Danielsen, E.M. Toxin-mediated effects on the innate mucosal defenses: implications for enteric vaccines. Infect. Immun. 2009, 77, 5206-5215.

76. Fan, E.; O’Neal, C.J.; Mitchell, D.D.; Robien, M.A.; Zhang, Z.; Pickens, J.C.; Tan, X.-J.; Korotkov, K.; Roach, C.; Krumm, B.; et al. Structural biology and structure-based inhibitor design of cholera toxin and heat-labile enterotoxin. Int. J. Med. Microbiol. 2004, 294, 217-223.

77. Lycke, N.; Bemark, M. Mucosal adjuvants and long-term memory development with special focus on CTA1-DD and other ADP-ribosylating toxins. Mucosal Immunol. 2010, 3, 556-566.

78. Iwata, M.; Hirakiyama, A.; Eshima, Y.; Kagechika, H.; Kato, C.; Song, S.-Y. Retinoic acid imprints gut-homing specificity on T cells. Immunity 2004, 21, 527-538.

79. Benson, M.J.; Pino-Lagos, K.; Rosemblatt, M.; Noelle, R.J. All-trans retinoic acid mediates enhanced $\mathrm{T}$ reg cell growth, differentiation, and gut homing in the face of high levels of co-stimulation. J. Exp. Med. 2007, 204, 1765-1774. 
80. Chen, Q.; Mosovsky, K.L.; Ross, A.C. Retinoic acid and alpha-galactosylceramide regulate the expression of costimulatory receptors and transcription factors responsible for B cell activation and differentiation. Immunobiology 2013, 218, 1477-1487.

81. Mora, J.R.; Pino-Lagos, K.; Rosemblatt, M.; Noelle, R.J. Generation of gut-homing IgA-secreting B cells by intestinal dendritic cells. Science 2006, 314, 1157-1160.

82. Pantazi, E.; Iwata, M.; Eksteen, B.; Song, S.-Y.; Junt, T.; Senman, B.; Otipoby, K.L.; Yokota, A.; Takeuchi, H.; Ricciardi-Castagnoli, P.; et al. Cutting Edge: Retinoic Acid Signaling in B Cells Is Essential for Oral Immunization and Microflora Composition. J. Immunol. 2015, 314, 1157-1160.

83. Hammerschmidt, S.I.; Friedrichsen, M.; Boelter, J.; Lyszkiewicz, M.; Kremmer, E.; Pabst, O.; Förster, R. Retinoic acid induces homing of protective $\mathrm{T}$ and $\mathrm{B}$ cells to the gut after subcutaneous immunization in mice. J. Clin. Invest. 2011, 121, 3051-3061.

84. Tan, X.; Sande, J.L.; Pufnock, J.S.; Blattman, J.N.; Greenberg, P.D. Retinoic acid as a vaccine adjuvant enhances CD8+ T cell response and mucosal protection from viral challenge. J. Virol. 2011, 85, 8316-8327.

85. Milpied, P.J.; McHeyzer-Williams, M.G. High-affinity IgA needs TH17 cell functional plasticity. Nat. Immunol. 2013, 14, 313-315.

86. Grassly, N.C.; Jafari, H.; Bahl, S.; Durrani, S.; Wenger, J.; Sutter, R.W.; Aylward, R.B. Mucosal immunity after vaccination with monovalent and trivalent oral poliovirus vaccine in India. $J$. Infect. Dis. 2009, 200, 794-801.

87. Jiang, V.; Jafari, H.; Bahl, S.; Durrani, S.; Wenger, J.; Sutter, R.W.; Aylward, R.B. Performance of rotavirus vaccines in developed and developing countries. Hum. Vaccin 2010, 6, 532-542.

88. Lopman, B.A.; Pitzer, V.E.; Sarkar, R.; Gladstone, B.; Patel, M.; Glasser, J.; Gambhir, M.; Atchison, C.; Grenfell, B.; Edmunds, W.J.; et al. Understanding reduced rotavirus vaccine efficacy in low socio-economic settings. PLOS ONE 2012, 7, e41720.

89. Levine, M.M. Immunogenicity and efficacy of oral vaccines in developing countries: Lessons from a live cholera vaccine. BMC Biol. 2010, 8, 129.

90. Mora, J.R.; Iwata, M.; von Andrian, U.H. Vitamin effects on the immune system: Vitamins A and D take centre stage. Nat. Rev. Immunol. 2008, 8, 685-698.

91. Kaufman, D.R.; De Calisto, J.; Simmons, N.L.; Cruz, A.N.; Villablanca, E.J.; Mora, J.R.; Barouch, D.H. Vitamin A deficiency impairs vaccine-elicited gastrointestinal immunity. J. Immunol. 2011, 187, 1877-1883.

92. Ibs, K.H.; Rink, L. Zinc-altered immune function. J. Nutr. 2003, 133 (Suppl. 1), 1452S-1456S.

93. Korpe, P.S.; Petri, W.A., Jr. Environmental enteropathy: Critical implications of a poorly understood condition. Trends Mol. Med. 2012, 18, 328-336.

94. Keusch, G.T.; Denno, D.M.; Black, R.E.; Duggan, C.; Guerrant, R.L.; Lavery, J.V.; Nataro, J.P.; Rosenberg, I.H.; Ryan, E.T.; Tarr, P.I.; et al. Environmental enteric dysfunction: Pathogenesis, diagnosis, and clinical consequences. Clin. Infect. Dis. 2014, 59 (Suppl. 4), S207-S212.

95. Majumder, P.P.; Sarkar-Roy, N.; Staats, H.; Ramamurthy, T.; Maiti, S.; Chowdhury, G.; Whisnant, C.C.; Narayanasamy, K.; Wagener, D.K. Genomic correlates of variability in immune response to an oral cholera vaccine. Eur. J. Hum. Genet. 2013, 21, 1000-1006. 
96. Stecher, B.; Chaffron, S.; Käppeli, R.; Hapfelmeier, S.; Freedrich, S.; Weber, T.C.; Kirundi, J.; Suar, M.; McCoy, K.D.; von Mering, C.; et al. Like will to like: abundances of closely related species can predict susceptibility to intestinal colonization by pathogenic and commensal bacteria. PLoS Pathog. 2010, 6, e1000711.

97. Moon, C.; Baldridge, M.T.; Wallace, M.A.; Burnham, C.-A.D.; Virgin, H.W.; Stappenbeck, T.S. Vertically transmitted faecal IgA levels determine extra-chromosomal phenotypic variation. Nature 2015, 521, 90-93.

98. Endt, K.; Maier, L.; Käppeli, R.; Barthel, M.; Misselwitz, B.; Kremer, M.; Hardt, W.-D. Peroral ciprofloxacin therapy impairs the generation of a protective immune response in a mouse model for Salmonella enterica serovar Typhimurium diarrhea, while parenteral ceftriaxone therapy does not. Antimicrob. Agents Chemother. 2012, 56, 2295-2304.

99. Uchiyama, R.; Chassaing, B.; Zhang, B.; Gewirtz, A.T. Antibiotic treatment suppresses rotavirus infection and enhances specific humoral immunity. J. Infect. Dis. 2014, 210, 171-182.

100. Praharaj, I.; John, S.M.; Bandyopadhyay, R.; Kang, G. Probiotics, antibiotics and the immune responses to vaccines. Philos. T. R. Soc. B 2015, 370, doi:10.1098/rstb.2014.0144.

101. Lindner, C.; Wahl, B.; Föhse, L.; Suerbaum, S.; Macpherson, A.J.; Prinz, I.; Pabst, O. Age, microbiota, and T cells shape diverse individual IgA repertoires in the intestine. J. Exp. Med. 2012, 209, 365-377.

102. Kole, A.; Maloy, K.J. Control of intestinal inflammation by interleukin-10. Curr. Top. Microbiol. Immunol. 2014, 380, 19-38.

103. Bollrath, J.; Powrie, F.M. Controlling the frontier: regulatory T-cells and intestinal homeostasis. Semin. Immunol. 2013, 25, 352-357.

104. Geuking, M.B.; Cahenzli, J.; Lawson, M.A.E.; Ng, D.C.K.; Slack, E.; Hapfelmeier, S.; McCoy, K.D.; Macpherson, A.J. Intestinal bacterial colonization induces mutualistic regulatory $\mathrm{T}$ cell responses. Immunity 2011, 34, 794-806.

105. Lindner, C.; Thomsen, I.; Wahl, B.; Ugur, M.; Sethi, M.K.; Friedrichsen, M.; Smoczek, A.; Ott, S.; Baumann, U.; Suerbaum, S.; et al. Diversification of memory B cells drives the continuous adaptation of secretory antibodies to gut microbiota. Nat. Immunol. 2015, 16, 880-888.

106. Akram, A.; Inman, R.D. Immunodominance: A pivotal principle in host response to viral infections. Clin. Immunol. 2012, 143, 99-115.

107. Buchbinder, S.P.; Mehrotra, D.V.; Duerr, A.; Fitzgerald, D.W.; Mogg, R.; Li, D.; Gilbert, P.B.; Lama, J.R.; Marmor, M.; del Rio, C.; et al. Efficacy assessment of a cell-mediated immunity HIV-1 vaccine (the Step Study): A double-blind, randomised, placebo-controlled, test-of-concept trial. Lancet 2008, 372, 1881-1893.

(C) 2015 by the authors; licensee MDPI, Basel, Switzerland. This article is an open access article distributed under the terms and conditions of the Creative Commons Attribution license (http://creativecommons.org/licenses/by/4.0/). 